\title{
An Unusual Complication of Hypertensive Hemorrhage - Delayed Oculomotor Palsy: Case Report and Literature Review
}

\author{
C. Dardis S. Sharfstein \\ Department of Neurology, SUNY Downstate Medical Center, Brooklyn, N.Y., USA
}

\section{Key Words}

Hypertensive hemorrhage - Aneurismal subarachnoid hemorrhage - Oculomotor palsy · MRI

\begin{abstract}
We present a case of oculomotor palsy due to hypertensive hemorrhage in the caudate nucleus, with intraventricular extension. To our knowledge, this is the only instance of this complication occurring due to hypertensive hemorrhage. Our patient initially developed headache at the time of her hemorrhage; 8 days later, she developed complete third nerve palsy, which showed improvement at follow-up 4 months later. This was due to tracking of blood into the perimesencephalic cistern. The presence of hemorrhage in the basal cisterns was not visible on the initial CT scans and highlights the role of MRI in evaluating the brainstem for the presence of blood products.
\end{abstract}

\section{Case Report}

The patient was a 45-year-old woman with a history of diabetes type 2, chronic kidney disease stage 4 and hypertension. She presented to hospital with a 3-day history of vomiting followed by dizziness and a diffuse headache. She had not used her antihypertensives for 3 days and her initial blood pressure was 195/100 mm Hg. Home medicines also included insulin, a statin, and a proton pump inhibitor. Her initial exam was remarkable for mild photophobia and neck stiffness. She was initially admitted with a working diagnosis of gastroenteritis causing dehydration and acute-on-chronic renal failure.

A CT of the head was performed on day 2 of admission due to persistent headache and neck stiffness; it showed hemorrhage in the left caudate lobe, with intraventricular extension (fig. 1 ).

Blood was visible in the lateral and 4th ventricles, although none was appreciated in the basal cisterns (fig. 2). Her initial MRI the same day confirmed the bleed. Although it was not initially remarked on, subsequent close inspection showed also perimesencephalic hemorrhage (fig. 3). MRAs of the head and neck were notable only for vasospasm of the distal left M2 artery. 
She remained stable until day 5 when she developed a complete oculomotor palsy on the left side. There was complete ptosis of the left eyelid. Her pupil was dilated to $6 \mathrm{~mm}$ and unreactive to light (vs. 3 $\mathrm{mm}$ and reactive on the right). The eye was deviated infero-laterally with almost no movement. Diplopia was present with the eye open, which was worst looking up and to the right. A repeat CT of the head showed resolution of the original hemorrhage. This made explanation of the palsy difficult. Her MRI and MRA of the head were unchanged. Given the imaging findings, we initially found this difficult to explain.

The localization was clearly in the subarachnoid space. The fascicle of the third nerve enters this space between the superior cerebellar and the posterior cerebral arteries, courses past the temporal lobe uncus and pierces the dura by the posterior clinoid process. As the ptosis was unilateral, it cannot have been nuclear. We would have expected involvement of other structures had the location been the brainstem or further anterior than the dura.

A number of possibilities concerned us: an aneurism of the posterior communicating artery compressing the nerve; ischemia due to her diabetes; migraine; or, given the presence of two lesions, CNS vasculitis. Ultimately, on closer review of the imaging, we determined the cause as hemorrhage tracking into the perimesencephalic cistern with involvement of the oculomotor nerve as it exited the brainstem.

Her stay was complicated: she developed sepsis, respiratory and renal failure requiring ventilation and hemodialysis. By day 37, she had recovered sufficiently to participate in physical therapy and was transferred to rehabilitation. When seen again in the clinic 4 months after the initial event, she had persistent oculomotor palsy, albeit much improved. Her diplopia had resolved.

\section{Discussion}

Blood in the subarachnoid space has been described as a cause of cranial nerve palsy in a number of other case reports. The majority of these occurred in the setting of aneurismal subarachnoid hemorrhage (SAH). Abducens palsy following hypertensive SAH has been reported, although it occurred in the setting of obstructive hydrocephalus requiring intraventricular drainage [1].

Oculomotor palsy has also been reported with subarachnoid bleeding due to other causes.

One case following spontaneous isolated perimesencephalic SAH [2] and 7 cases due to idiopathic SAH have been reported [3]. Recurrent episodes following pituitary hemorrhage, presumably with subarachnoid extension have been noted [4]. Both oculomotor and trochlear palsies have been reported following rupture of ACA and MCA aneurisms [5, 6]. Abducens palsy following aneurismal SAH has also been documented [7]. In all these cases, the diplopia began at the time of the headache, except one which began hours later and was suspected to be due to rebleeding of an aneurism.

In the largest case series on abducens palsy following aneurismal SAH (occurring in 6 of 101 cases), it occurred between 3 and 14 days after onset of SAH, with an average of 7.3 days. The authors found the size of the prepontine clot to be the best predictor of the presence of palsy [8]. This argues for a primarily mechanical effect. Local inflammatory infiltrates around the origin of the nerve or nearby vasospasm have been suggested also; this appears less likely as other nearby structures are uninvolved in such cases.

The delay in onset of palsy is of particular interest in our case. The fact that it took 8 days from symptom onset is consistent with the above series of abducens palsies. It suggests a role for clot organization, leading to nerve compression. We are not aware of 
any studies addressing the time course of clot retraction in CSF. CSF does contain tissue factor so we would expect a thrombus therein to behave similarly to a venous thrombus. An experimental study in rabbits showed the appearance of completely organized thrombi by around 10 days [9]. In a similar study on the arterial system in pigs, organization was present by this time, with signs of replacement of fibrin by contractile cellular elements (fibroblasts and smooth muscle cells) [10].

It may be argued that coincidental microvascular ischemia in the fascicle of the third nerve was the cause of the lesion in our patient. Classically, this spares the pupil and complete resolution within 3 months is the rule. However, the largest case series on oculomotor palsy, with 1,400 patients, showed that $50 \%$ had pupillary involvement [3]. Only $3 \%$ of cases due to diabetes had anisocoria of $>2 \mathrm{~mm}$ as did our patient, and in only $66 \%$ of cases was the diabetes the cause of the palsy. Again complete resolution, albeit slow, was the rule. Another series showed pupillary involvement in $38 \%$ of cases; however, none of these pupils were reported to be fully dilated and unreactive [11]. Thus, in our patient, the degree of anisocoria and the persistence of the deficit made this much less likely.

A fully dilated, unreactive pupil has been estimated to occur in up to $70 \%$ of patients with aneurismal compression of the oculomotor nerve [12]. In the absence of diabetes or hypertension, particular attention to the possibility of compression or infiltration has been suggested for such patients. Given our patient's normal MRA findings, conventional angiography would not have been expected to yield relevant findings. Ophthalmoplegic migraine was a consideration; however, we would have expected resolution of the palsy with the headache.

This case highlights the importance of considering SAH as a cause of oculomotor palsy. This should always be kept in mind in the differential diagnosis, particularly in the setting of new headache with neck stiffness, even in patients with diabetes and hypertension. It also highlights the added value of MRI in demonstrating lesions in the brainstem, including the perimesencephalon. 


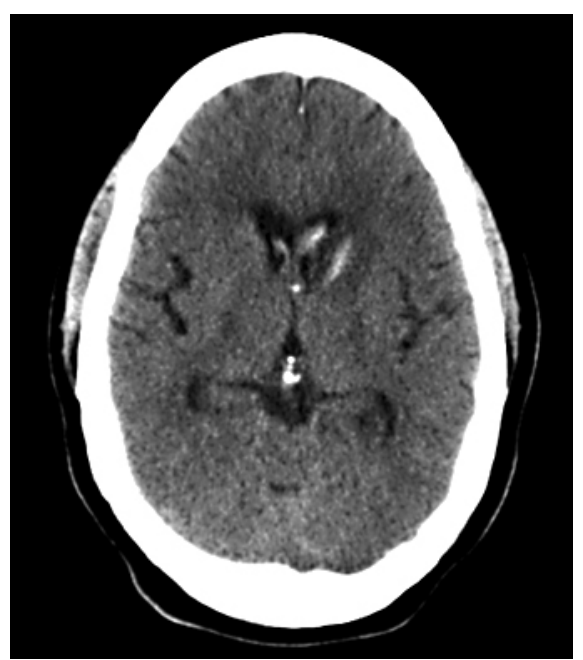

Fig. 1. CT of the head on day 3 showing hemorrhage in the left caudate nucleus, with extension into the right lateral ventricle.

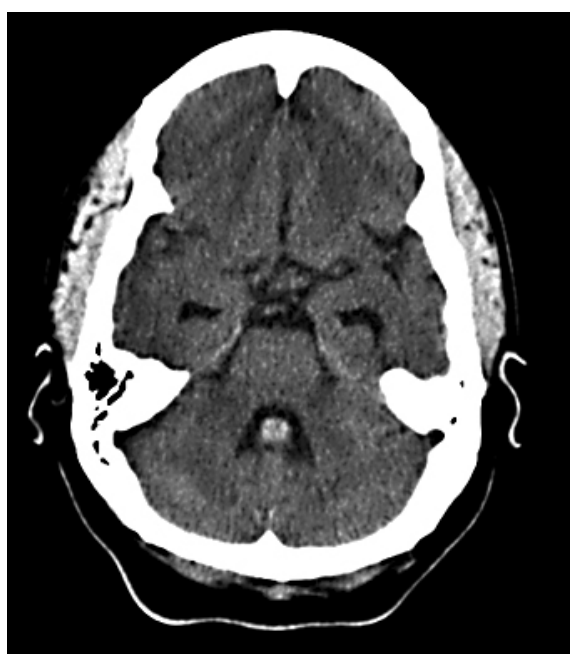

Fig. 2. CT of the head on day 3 showing tracking of blood into the 4 th ventricle, with enlargement of the 4 th ventricle. No blood is visible in the basal cisterns. 


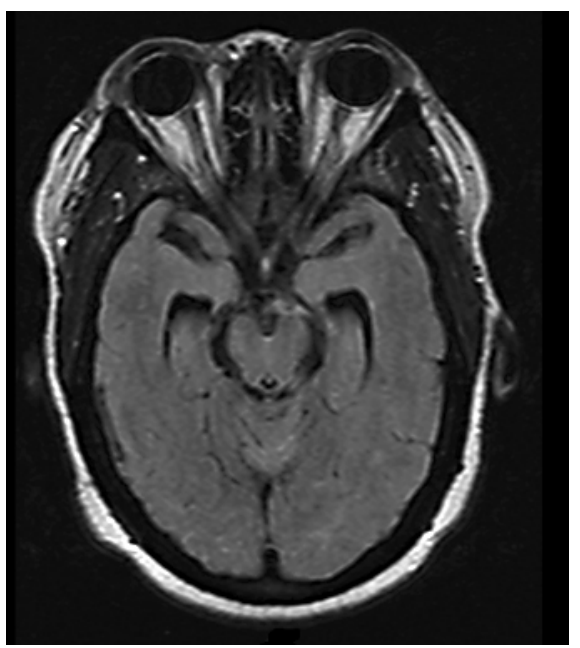

Fig. 3. MRI of the brain (FLAIR sequence) on day 3 showing high signal at the left perimesencephalic cistern. This area was bright also on the T1 sequence. This is typical for late subacute hemorrhage, indicating the presence of free (extracellular) methemoglobin.

\section{References}

1 Ayberk G, Ozveren MF, Yildirim T, Ercan K, Cay EK, Koçak A: Review of a series with abducens nerve palsy. Turk Neurosurg 2008;18:366-373.

2 Kamat AA, Tizzard S, Mathew B: Painful third nerve palsy in a patient with perimesencephalic subarachnoid haemorrhage. Br J Neurosurg 2005;19:247-250.

3 Keane JR: Third nerve palsy: analysis of 1,400 personally-examined inpatients. Can J Neurol Sci 2010;37:662670 .

4 Mohanty S: Recurrent oculomotor palsy due to haemorrhage in pituitary adenoma. Postgrad Med J 1980;56:54-56.

5 White JB, Layton KF, Cloft HJ: Isolated third nerve palsy associated with a ruptured anterior communicating artery aneurysm. Neurocrit Care 2007;7:260-262.

6 Kim SC, Chung J, Lim YC, Shin YS: Oculomotor nerve palsy associated with rupture of middle cerebral artery aneurysm. J Korean Neurosurg Soc 2009;45:240-242.

7 Göksu E, Akyüz M, Gürkanlar D, Tuncer R: Bilateral abducens nerve palsy following ruptured anterior communicating artery aneurysm: report of 2 cases. Neurocirugia (Astur) 2007;18:420-422.

8 Munakata A, Ohkuma H, Nakano T, Shimamura N: Abducens nerve pareses associated with aneurysmal subarachnoid hemorrhage. Incidence and clinical features. Cerebrovasc Dis 2007;24:516-519.

-9 Jorgensen L: Experimental platelet and coagulation thrombi. A histological study of arterial and venous thrombi of varying age in untreated and heparinized rabbits. Acta Pathol Microbiol Scand 1964;62:189-223.

-10 Jorgensen L, Rowsell HC, Hovig T, Mustard JF: Resolution and organization of platelet-rich mural thrombi in carotid arteries of swine. Am J Pathol 1967;51:681-719.

11 Jacobson DM: Pupil involvement in patients with diabetes-associated oculomotor nerve palsy. Arch Ophthalmol 1998;116:723-727.

12 Keane JR: Aneurysms and third nerve palsies. Ann Neurol 1983;14:696-669. 